\title{
www.czasopisma.pan.pl \\ Dual Phase Lag Model of Melting Process in Domain of Metal Film Subjected to an External Heat Flux
}

\author{
B. Mochnacki ${ }^{\text {a, *, }}$ M. Ciesielski ${ }^{\text {b }}$ \\ ${ }^{a}$ University of Occupational Safety Management in Katowice, Bankowa 8, 40-007 Katowice, Poland \\ ${ }^{\mathrm{b}}$ Czestochowa University of Technology, Dabrowskiego 69, 42-200 Czestochowa, Poland \\ *Corresponding author: e-mail: bmochnacki@wszop.edu.pl
}

Received 30.05.2016; accepted in revised form 14.07.2016

\begin{abstract}
Heating process in the domain of thin metal film subjected to a strong laser pulse are discussed. The mathematical model of the process considered is based on the dual-phase-lag equation (DPLE) which results from the generalized form of the Fourier law. This approach is, first of all, used in the case of micro-scale heat transfer problems (the extremely short duration, extreme temperature gradients and very small geometrical dimensions of the domain considered). The external heating (a laser action) is substituted by the introduction of internal heat source to the DPLE. To model the melting process in domain of pure metal (chromium) the approach basing on the artificial mushy zone introduction is used and the main goal of investigation is the verification of influence of the artificial mushy zone 'width' on the results of melting modeling. At the stage of numerical modeling the author's version of the Control Volume Method is used. In the final part of the paper the examples of computations and conclusions are presented.
\end{abstract}

Keywords: Theoretical basis of foundry processes, Solidification process, Micro-scale heat transfer, Dual phase lag model, Control volume method

\section{Introduction}

The base for the macroscopic model of alloys solidification or melting is the well known Fourier equation with an additional term (the source function) controlling the evolution of latent heat [1-3]. The assumption that the local and temporary value of solid state volumetric fraction is the known temperature-dependent function allows one to transform the basic energy equation, and then the parameter called 'a substitute thermal capacity' appears (e.g. [2]). Such an approach is known as a one domain method or a fixed domain method. It turned out that the similar transformation can be done in the case of dual-phase-lag equation [4]. The one domain method describes the melting or solidification processes in the case of materials for which the phase transition proceeds in the interval of temperature (e.g. binary alloys). In the case of pure metal solidification the solidification point $T^{*}$ has to be replaced by a certain temperature interval $\left[T^{*}-\Delta T, T^{*}+\Delta T\right]$ and in this way the artificial mushy zone is introduced. Next, for this interval the substitute thermal capacity should be defined [1-3]. In literature the macro models of melting (solidification) are widely discussed (e.g. [1-3], [5-7]), while the number of papers concerning the micro-scale models is rather small, among others $[4,8,9,10]$. In this paper the analysis of relationships between width of the border temperatures interval $T^{*}-\Delta T$ and $T^{*}+\Delta T$ and the results of numerical solution of the task discussed is done. The similar research concerning the macro models of melting (solidification) process has been presented by Szopa in [5]. 


\section{Mathematical model}

The dual phase lag equation results from the generalized form of the Fourier law containing two lag times, in particular the relaxation and to thermalization times $[11,12,13]$

$\mathbf{q}\left(r, z, t+\tau_{q}\right)=-\lambda \nabla T\left(r, z, t+\tau_{T}\right)$

where $\tau_{q}$ and $\tau_{T}$ are the phase lags (relaxation and thermalization times), while $\mathbf{q}$ is a heat flux, $\lambda$ is a thermal conductivity, $r, z$ are the geometrical co-ordinates (axially-symmetrical problem is considered), $t$ is a time.

Using the Taylor series expansions, the following first-order approximation of equation (1) is obtained

$\mathbf{q}(r, z, t)+\tau_{q} \frac{\partial \mathbf{q}(r, z, t)}{\partial t}=-\lambda\left[\nabla T(r, z, t)+\tau_{T} \frac{\partial \nabla T(r, z, t)}{\partial t}\right]$

Introducing this formula to the well known diffusion equation after the mathematical manipulations one has

$$
\begin{gathered}
c\left[\frac{\partial T(r, z, t)}{\partial t}+\tau_{q} \frac{\partial^{2} T(r, z, t)}{\partial t^{2}}\right]=\nabla[\lambda \nabla T(r, z, t)]+ \\
\tau_{T} \frac{\partial\{\nabla[\lambda \nabla T(r, z, t)]\}}{\partial t}+Q(r, z, t)+\tau_{q} \frac{\partial Q(r, z, t)}{\partial t}
\end{gathered}
$$

Here $c$ is a volumetric specific heat of material, $Q$ is the capacity of internal heat sources. In the case considered the function $Q$ is the sum of two components. The first of them is connected with the laser beam action and according to [11] the suitable source term is of the form

$$
Q_{L}(r, z, t)=\sqrt{\frac{4 \ln 2}{\pi}}(1-R) \frac{I_{0}}{\delta t_{p}} \exp \left[-\frac{r^{2}}{r_{D}^{2}}-\frac{z}{\delta}-4 \ln 2 \frac{\left(t-2 t_{p}\right)^{2}}{t_{p}^{2}}\right]
$$

where $I_{0}\left[\mathrm{~J} / \mathrm{m}^{2}\right]$ is a laser intensity, $t_{p}[\mathrm{~s}]$ is a characteristic time of laser pulse, $\delta[\mathrm{m}]$ is an optical penetration depth, $R$ is a reflectivity of irradiated surface, $r_{D}[\mathrm{~m}]$ is a laser beam radius. The derivative of $Q_{L}$ with respect to time (equation (4)) can be found analytically.

The second source function is connected with the evolution of latent heat. The well known form of this component is the following

$Q_{S}(r, z, t)=L \frac{\partial f_{S}(r, z, t)}{\partial t}=L \frac{\mathrm{d} f_{S}(T)}{\mathrm{d} T} \frac{\partial T(r, z, t)}{\partial t}$

where $L$ is a volumetric latent heat, $f_{S}$ is a volumetric solid state fraction at the neighborhood of point considered.

Let us assume that the function $f_{S}$ in the interval $\left[T^{*}-\Delta T\right.$, $\left.T^{*}+\Delta T\right]$ is a linear one, this means

$$
f_{S}(r, z, t)=\frac{T^{*}+\Delta T-T(r, z, t)}{2 \Delta T}
$$

One can see that outside the interval between the border temperatures the function $f_{S}$ takes the values 1 and 0 (solid and liquid states). In the paper [4] was shown that the source term controlling the evolution of latent heat can be written as follows

$$
\begin{aligned}
& Q_{S}(r, z, t)+\tau_{q} \frac{\partial Q_{S}(r, z, t)}{\partial t}= \\
& L \frac{\mathrm{d} f_{S}(T)}{\mathrm{d} T}\left[\frac{\partial T(r, z, t)}{\partial t}+\tau_{q} \frac{\partial^{2} T(r, z, t)}{\partial t^{2}}\right]
\end{aligned}
$$

and finally one obtains the DPLE in the form

$$
\begin{gathered}
C\left[\frac{\partial T(r, z, t)}{\partial t}+\tau_{q} \frac{\partial^{2} T(r, z, t)}{\partial t^{2}}\right]=\nabla[\lambda \nabla T(r, z, t)]+ \\
\tau_{T} \frac{\partial\{\nabla[\lambda \nabla T(r, z, t)]\}}{\partial t}+Q_{L}(r, z, t)+\tau_{q} \frac{\partial Q_{L}(r, z, t)}{\partial t}
\end{gathered}
$$

where

$$
C=\left\{\begin{array}{lc}
c_{L} & T>T^{*}+\Delta T \\
c_{M}+\frac{L}{2 \Delta T} & T^{*}-\Delta T \leq T \leq T^{*}+\Delta T \\
c_{S} & T<T^{*}-\Delta T
\end{array}\right.
$$

while $c_{L}, c_{S}$ are the volumetric specific heats of liquid and solid state, $c_{M}$ is (for example) the arithmetic mean of $c_{L}$ and $c_{S}$.

The no-flux boundary condition given on the external surface of the system is the following $[4,14]$

$-\lambda\left[\mathbf{n} \cdot \nabla T(r, z, t)+\tau_{T} \frac{\partial[\mathbf{n} \cdot \nabla T(r, z, t)]}{\partial t}\right]=0$

where $\mathbf{n} \cdot \nabla T(r, z, t)$ is the temperature derivative in the normal direction.

The initial conditions (the initial temperature of domain and the initial heating rate) are also given

$t=0: \quad T(r, z, 0)=T_{0},\left.\quad \frac{\partial T(r, z, t)}{\partial t}\right|_{t=0}=0$

where $T_{0}$ is the initial temperature of domain.

\section{Control Volume Method}

At the stage of numerical computations the Control Volume Mehod (CVM) in the version proposed by Ciesielski and Mochnacki [14] is applied. As is well known, the CVM algorithm allows one to find the transient temperature field at the set of nodes corresponding to the central points of control volumes. The nodal temperatures can be found on the basis of energy balances 
for the successive volumes. The energy balances corresponding to heat exchange between the analyzed control volume and adjoining control volumes results from the integration of equation (8) with respect to control volume and time. In Figure 1 the cylindrical, axially symmetrical domain and its discretization is shown.

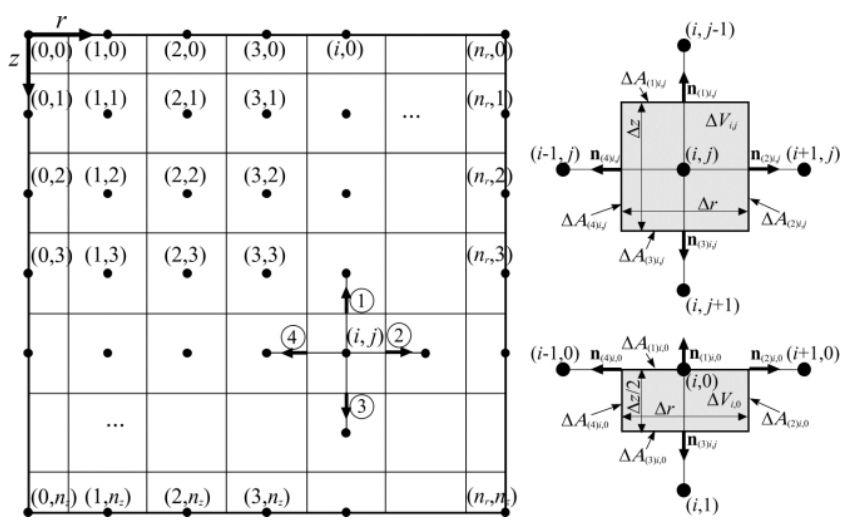

Fig. 1. The discretization of domain

So, the energy equation (8) should be integrated over the control volume $\Omega_{i, j}$

$$
\begin{aligned}
\int_{\Omega_{i, j}} c & \left(\frac{\partial T(r, z, t)}{\partial t}+\tau_{q} \frac{\partial^{2} T(r, z, t)}{\partial t^{2}}\right) d \Omega \\
= & \int_{\Omega_{i, j}}\left(\nabla \cdot[\lambda \nabla T(r, z, t)]+\tau_{T} \frac{\partial \nabla \cdot[\lambda \nabla T(r, z, t)]}{\partial t}\right) d \Omega \\
& +\int_{\Omega_{i, j}}\left(Q(r, z, t)+\tau_{q} \frac{\partial Q(r, z, t)}{\partial t}\right) d \Omega
\end{aligned}
$$

Integration of the left hand side of equation (12) gives

$$
\begin{gathered}
\int_{\Omega_{i, j}} c\left(\frac{\partial T(r, z, t)}{\partial t}+\tau_{q} \frac{\partial^{2} T(r, z, t)}{\partial t^{2}}\right) d \Omega \approx \\
c_{i, j}\left(\frac{\mathrm{d} T_{i, j}}{\mathrm{~d} t}+\tau_{q} \frac{\mathrm{d}^{2} T_{i, j}}{\mathrm{~d} t^{2}}\right) \Delta V_{i, j}
\end{gathered}
$$

The source term in Eq. (8) is treated in a similar way. Applying the divergence theorem to the term determining heat conduction between the volume $\Omega_{i, j}$ bounded by the surfaces $\Delta A_{i, j}$ and its neighbourhoods one obtains

$$
\begin{gathered}
\int_{\Omega_{i, j}}\left(\nabla \cdot[\lambda \nabla T(r, z, t)]+\tau_{T} \frac{\partial \nabla \cdot[\lambda \nabla T(r, z, t)]}{\partial t}\right) d \Omega \\
\quad=\int_{A_{i, j}}\left(\mathbf{n} \cdot \lambda\left(\nabla T(r, z, t)+\tau_{T} \frac{\partial \nabla T(r, z, t)}{\partial t}\right)\right) d A
\end{gathered}
$$

$$
\int_{A_{i, j}}\left(\mathbf{n} \cdot \lambda\left(\nabla T(r, z, t)+\tau_{T} \frac{\partial \nabla T(r, z, t)}{\partial t}\right)\right) d A=\sum_{k=1}^{4}\left(q_{k}\right)_{i, j}\left(\Delta A_{k}\right)_{i, j}
$$

where

$$
\begin{aligned}
& \left(q_{1}\right)_{i, j}= \begin{cases}\left(\lambda_{1}\right)_{i, j}\left(\frac{T_{i, j-1}-T_{i, j}}{\Delta z}+\tau_{T} \frac{\mathrm{d}}{\mathrm{d} t}\left(\frac{T_{i, j-1}-T_{i, j}}{\Delta z}\right)\right) & \text { if } j>0 \\
0 & \text { if } j=0\end{cases} \\
& \left(q_{2}\right)_{i, j}= \begin{cases}\left(\lambda_{2}\right)_{i, j}\left(\frac{T_{i+1, j}-T_{i, j}}{\Delta r}+\tau_{T} \frac{\mathrm{d}}{\mathrm{d} t}\left(\frac{T_{i+1, j}-T_{i, j}}{\Delta r}\right)\right) & \text { if } i<n_{r} \\
0 & \text { if } i=n_{r}\end{cases} \\
& \left(q_{3}\right)_{i, j}= \begin{cases}\left(\lambda_{3}\right)_{i, j}\left(\frac{T_{i, j+1}-T_{i, j}}{\Delta z}+\tau_{T} \frac{\mathrm{d}}{\mathrm{d} t}\left(\frac{T_{i, j+1}-T_{i, j}}{\Delta z}\right)\right) & \text { if } j<n_{z} \\
0 & \text { if } j=n_{z}\end{cases} \\
& \left(q_{4}\right)_{i, j}= \begin{cases}\left(\lambda_{4}\right)_{i, j}\left(\frac{T_{i-1, j}-T_{i, j}}{\Delta r}+\tau_{T} \frac{\mathrm{d}}{\mathrm{d} t}\left(\frac{T_{i-1, j}-T_{i, j}}{\Delta r}\right)\right) & \text { if } i>0 \\
0 & \text { if } i=0\end{cases}
\end{aligned}
$$

and $\left(\lambda_{k}\right)_{i, j}$ are the harmonic mean thermal conductivities between two central points of adjoining control volumes. After the introduction of all discrete terms into equation (12) one obtains

$$
\begin{gathered}
c_{i, j}\left(\frac{\mathrm{d} T_{i, j}}{\mathrm{~d} t}+\tau_{q} \frac{\mathrm{d}^{2} T_{i, j}}{\mathrm{~d} t^{2}}\right) \Delta V_{i, j}=\sum_{k=1}^{4}\left(q_{k}\right)_{i, j}\left(\Delta A_{k}\right)_{i, j}+ \\
\left(Q_{i, j}+\tau_{q} \frac{\mathrm{d} Q_{i, j}}{\mathrm{~d} t}\right) \Delta V_{i, j}
\end{gathered}
$$

The second stage of CVM is the integration of equation (20) with respect to time and then the energy balances written in the convention of 'explicit' scheme (for the transition $t^{f-1}$ to $t^{f}, f=$ $2, \ldots, F$ ) take the form

$$
\begin{gathered}
c_{i, j}^{f-1}\left(\frac{T_{i, j}^{f}-T_{i, j}^{f-1}}{\Delta t}+\tau_{q} \frac{T_{i, j}^{f}-2 T_{i, j}^{f-1}+T_{i, j}^{f-2}}{(\Delta t)^{2}}\right) \Delta V_{i, j}= \\
\sum_{k=1}^{4}\left(q_{k}\right)_{i, j}^{f-1}\left(\Delta A_{k}\right)_{i, j}+\left(Q_{i, j}^{f}+\tau_{q} \frac{Q_{i, j}^{f}-Q_{i, j}^{f-1}}{\Delta t}\right) \Delta V_{i, j}
\end{gathered}
$$

where $\Delta t$ denotes a time step. The heat fluxes appearing in the last equation (after non complex mathematical manipulations) can be written as follows

$$
\left(q_{1}\right)_{i, j}^{f-1}= \begin{cases}\frac{T_{i, j-1}^{f-1}-T_{i, j}^{f-1}}{\left(R_{1}\right)_{i, j}^{f-1}}+\tau_{T}\left(\frac{T_{i, j-1}^{f-1}-T_{i, j-1}^{f-2}}{\left(R_{1}\right)_{i, j}^{f-1} \Delta t}-\frac{T_{i, j}^{f-1}-T_{i, j}^{f-2}}{\left(R_{1}\right)_{i, j}^{f-1} \Delta t}\right) & j>0 \\ 0 & j=0\end{cases}
$$

and next this term can be written in the form 


$$
\begin{aligned}
& \left(q_{2}\right)_{i, j}^{f-1}= \begin{cases}\frac{T_{i+1, j}^{f-1}-T_{i, j}^{f-1}}{\left(R_{2}\right)_{i, j}^{f-1}}+\tau_{T}\left(\frac{T_{i+1, j}^{f-1}-T_{i+1, j}^{f-2}}{\left(R_{2}\right)_{i, j}^{f-1} \Delta t}-\frac{T_{i, j}^{f-1}-T_{i, j}^{f-2}}{\left(R_{2}\right)_{i, j}^{f-1} \Delta t}\right) & i<n_{r} \\
0 & i=n_{r}\end{cases} \\
& \left(q_{3}\right)_{i, j}^{f-1}= \begin{cases}\frac{T_{i, j+1}^{f-1}-T_{i, j}^{f-1}}{\left(R_{3}\right)_{i, j}^{f-1}}+\tau_{T}\left(\frac{T_{i, j+1}^{f-1}-T_{i, j}^{f-1}}{\left(R_{3}\right)_{i, j}^{f-1} \Delta t}-\frac{T_{i, j}^{f-1}-T_{i, j}^{f-2}}{\left(R_{3}\right)_{i, j}^{f-1} \Delta t}\right) & j<n_{z} \\
0 & j=n_{z}\end{cases} \\
& \left(q_{4}\right)_{i, j}^{f-1}= \begin{cases}\frac{T_{i+1, j}^{f-1}-T_{i, j}^{f-1}}{\left(R_{4}\right)_{i, j}^{f-1}}+\tau_{T}\left(\frac{T_{i+1, j}^{f-1}-T_{i+1, j}^{f-2}}{\left(R_{4}\right)_{i, j}^{f-1} \Delta t}-\frac{T_{i, j}^{f-1}-T_{i, j}^{f-2}}{\left(R_{4}\right)_{i, j}^{f-1} \Delta t}\right) & i>0 \\
0 & i=0\end{cases}
\end{aligned}
$$

The acceptation of the 'directional' thermal conductivities as the harmonic means of the nodal values causes that in the formulas (30) determining heat fluxes $q$ the thermal resistances between the neighbouring nodes appear [15], in particular for the internal nodes

$$
\begin{aligned}
& \left(R_{1}\right)_{i, j}^{f-1}=\frac{0.5 \Delta z}{\lambda_{i, j}^{f-1}}+\frac{0.5 \Delta z}{\lambda_{i, j-1}^{f-1}} \\
& \left(R_{2}\right)_{i, j}^{f-1}=\frac{0.5 \Delta r}{\lambda_{i, j}^{f-1}}+\frac{0.5 \Delta r}{\lambda_{i+1, j}^{f-1}} \\
& \left(R_{3}\right)_{i, j}^{f-1}=\frac{0.5 \Delta z}{\lambda_{i, j}^{f-1}}+\frac{0.5 \Delta z}{\lambda_{i, j+1}^{f-1}} \\
& \left(R_{4}\right)_{i, j}^{f-1}=\frac{0.5 \Delta r}{\lambda_{i, j}^{f-1}}+\frac{0.5 \Delta r}{\lambda_{i-1, j}^{f-1}}
\end{aligned}
$$

while for the boundary ones the thermal resistance in direction 'to the boundary' can be assuned as a very big number, e.g. $10^{10}$.

The final form of the CVM equations can be written in the form

$$
\begin{aligned}
T_{i, j}^{f}= & \frac{\Delta t+2 \tau_{q}}{\Delta t+\tau_{q}} T_{i, j}^{f-1}-\frac{\tau_{q}}{\Delta t+\tau_{q}} T_{i, j}^{f-2}+ \\
& \frac{(\Delta t)^{2}}{\left(\Delta t+\tau_{q}\right) c_{i, j}^{f-1}} \sum_{k=1}^{4}\left(q_{k}\right)_{i, j}^{f-1}\left(\Phi_{k}\right)_{i, j}+\frac{\Delta t}{c_{i, j}^{f-1}}\left(Q_{i, j}^{f}-\frac{\tau_{q}}{\Delta t+\tau_{q}} Q_{i, j}^{f-1}\right)
\end{aligned}
$$

At the same time $\left(\Phi_{k}\right)_{i, j}=\left(\Delta A_{k}\right)_{i, j} / \Delta V_{i, j}$. The formula (30) shows that in the case of hyperbolic equations three-level CVM approximation must be used, in other words, in order to determine the temperature $T_{i, j}^{f}$ (time level $f$ ) the nodal temperatures corresponding to time levels $f-1$ and $f-2$ must be known. Using the initial conditions (11), the following starting point should be accepted: $T_{i, j}^{0}=T_{i, j}^{1}=T_{0}$.

Because the explicit scheme has been used, therefore the stability conditions should be fulfilled. The authors found that this condition for the task considered is the following

$$
\Delta t+2 \tau_{q}-\Delta t\left(\Delta t+\tau_{T}\right) W_{i, j}^{f-1} \geq 0
$$

The coefficient $W$ is equal to

$$
W_{i, j}^{f-1}=\frac{1}{c_{i, j}^{f-1}}\left[\frac{\left(\Phi_{1}\right)_{i, j}}{\left(R_{1}\right)_{i, j}^{f-1}}+\frac{\left(\Phi_{2}\right)_{i, j}}{\left(R_{2}\right)_{i, j}^{f-1}}+\frac{\left(\Phi_{3}\right)_{i, j}}{\left(R_{3}\right)_{i, j}^{f-1}}+\frac{\left(\Phi_{4}\right)_{i, j}}{\left(R_{4}\right)_{i, j}^{f-1}}\right]
$$

From inequality (32) one can determine the proper time step $\Delta t$

$$
\Delta t \in\left(0, \frac{1+\sqrt{\left(\tau_{T} W_{\max }-1\right)^{2}+8 \tau_{q} W_{\max }}}{2 W_{\max }}-\frac{\tau_{T}}{2}\right]
$$

where $W_{\max }=\max _{(i, j), f}\left(W_{i, j}^{f-1}\right)$.

\section{Examples of computations}

Numerical simulation of a thermal process proceeding in fomain of thin metal film subjected to the short laser pulse has been done for the cylindrical domain with dimensions $Z=$ $100 \mathrm{~nm}, R=100 \mathrm{~nm}-$ Figure $2[14]$.

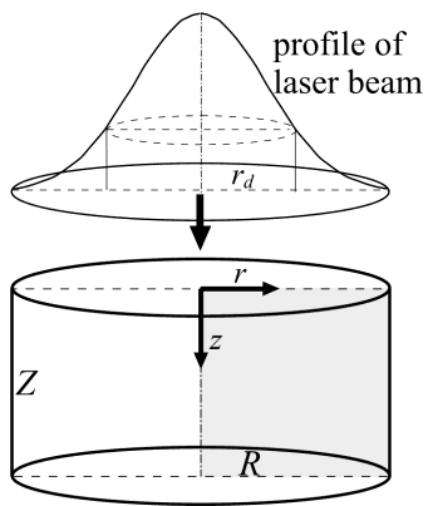

Fig. 2. Micro-domain considered

Thermophysical parameters of material (chromium) are equal to $\lambda=93 \mathrm{~W} /(\mathrm{mK}), c_{S}=c_{L}=3.2148 \cdot 10^{6} \mathrm{~J} /\left(\mathrm{m}^{3} \mathrm{~K}\right), \tau_{q}=0.136 \mathrm{ps}$, $\tau_{T}=7.86 \mathrm{ps}[16], L=2904 \cdot 10^{6} \mathrm{~J} / \mathrm{m}^{3}$, solidification point $T^{*}=$ $1857^{\circ} \mathrm{C}, \Delta T=3,5,8 \mathrm{~K}$, respectively. The parameters of the belltype laser pulse are following: $r_{d}=50 \mathrm{~nm}, I_{0}=3000 \mathrm{~J} / \mathrm{m}^{2}, R_{f}=$ $0.93, \delta=15.3 \mathrm{~nm}, t_{p}=100 \mathrm{fs}$. The initial temperature of the metal is $T_{0}=20^{\circ} \mathrm{C}$. The mesh steps used in this example: $\Delta z=10^{-9} \mathrm{~m}$, $\Delta r=10^{-9} \mathrm{~m}$ and the time step $\Delta t=10^{-17} \mathrm{~s}$.

The results of computations presented in Figures 3-5 illustrate the temperature histories at three selected nodes in the domain for the different values of $\Delta T$. In Figure 6 the temperature field for time 0.3 ps is shown. 


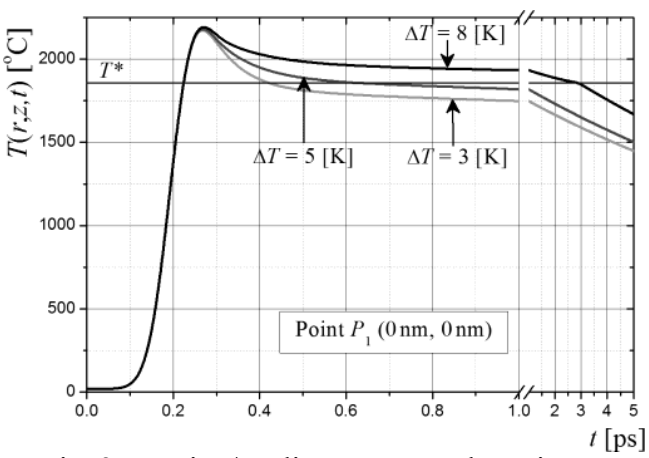

Fig. 3. Heating/cooling curves at the point $P_{1}$

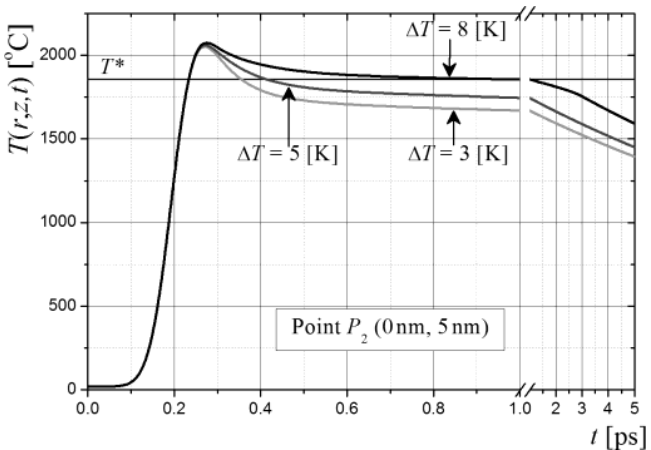

Fig. 4. Heating/cooling curves at the point $P_{2}$

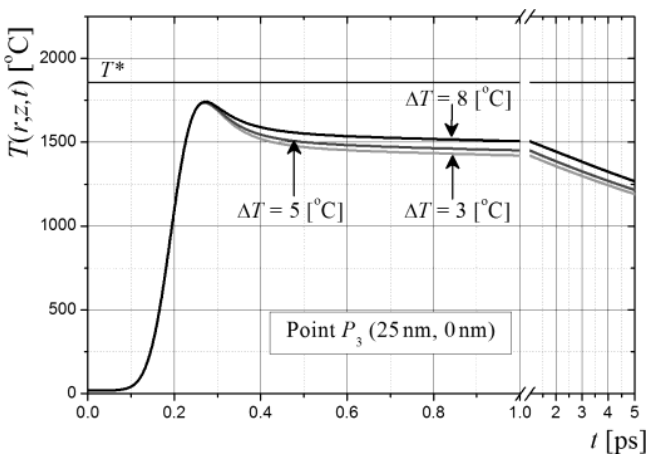

Fig. 5. Heating/cooling curves at the point $P_{3}$

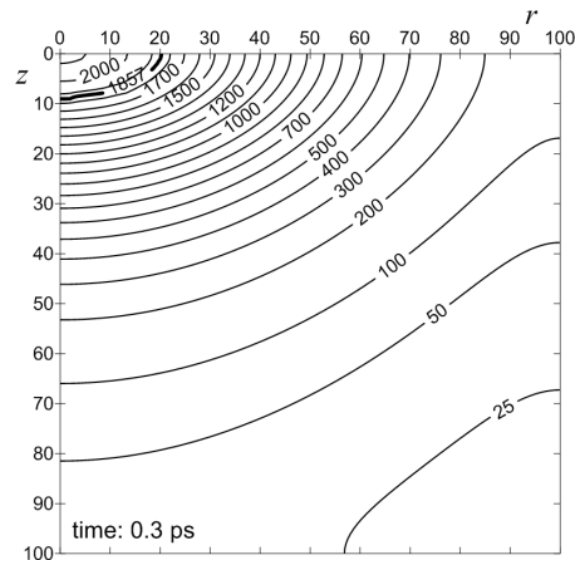

Fig. 6. Temperature field for time $0.3 \mathrm{ps}(\Delta T=5[\mathrm{~K}])$

\section{Final remarks}

The authors solved the problem of pure metal melting and resolidification by the introduction of artificial mushy zone to the mathematical model of the process. At the stage of numerical computations the different values of $\Delta T$ (a width of mushy zone) have been taken into account. The differences of numerical solutions are small, but visible. The same example has been also solved using the Fourier equation (both $\tau_{q}$ and $\tau_{T}$ are equal to zero). It turned out that for this model the heating process proceeds more intensively and for $I_{0}=3000 \mathrm{~J} / \mathrm{m}^{2}$ the metal temperature (close to the point $P_{1}$ ) exceeds the evaporation point. The results closer to the presented above have been obtained for $I_{0}=2600 \mathrm{~J} / \mathrm{m}^{2}$. The typical decrease of heating/cooling rate close to the solidification point (easily visible in the case of macro-scale solidification models - e.g. [5]) is here almost unnoticeable. It results from essential differences between capacity of internal heat source resulting from the laser action and the capacity of heat source resulting from the melting or solidification processes.

\section{Acknowledgement}

The paper is a part of Research Project 2015/19/B/ST8/01101 sponsored by NCN.

\section{References}

[1] Mochnacki, B. \& Majchrzak, E. (2010). Numerical modeling of casting solidification using generalized finite difference method. Materials Science Forum. 638-642, 2676-2681.

[2] Mochnacki, B. (2012). Definition of alloy substitute thermal capacity using the simple macrosegregation models. Archives of Foundry Engineering. 19(4), 113-116.

[3] Mochnacki, B. (2011). Computational simulations and applications. Numerical modeling of solidification process (Chapter 24), Ed. Jianping Zhu, INTECH, 513-542.

[4] Majchrzak, E. \& Mochnacki, B. (2016). Modeling of melting and resolidification in domain of metal film subjected to a laser pulse. Archives of Foundry Engineering. 16(1), 41-44.

[5] Szopa, R. (2015). Numerical modeling of pure metal solidification using the one domain approach. Journal of Applied Mathematics and Computational Mechanics. 14(3), 28-34.

[6] Bondarenko, V.I., Bilousov, V.V., Nedopekin, F.V. \& Shalapko, J.I. (2015). The mathematical model of hydrodynamics and heat and mass transfer at formation of steel ingots and castings. Archives of Foundry Engineering. 15(1), 13-16.

[7] Ivanova, A.A. (2012). Calculation of phase change boundary position in continuous casting. Archives of Foundry Engineering. 13(4), 57-62.

[8] Mochnacki, B., Majchrzak, E. (2016). Chapter 86: Numerical modeling of biological tissue freezing process using the DualPhase-Lag Equation, Advances in Mechanics: Theoretical, Computational and Interdisciplinary Issues, Proceedings of the 3rd Polish Congress of Mechanics (PCM) and 21st 
International Conference on Computer Methods in Mechanics (CMM), CRC Press, 413-418.

[9] Majchrzak, E. \& Dziatkiewicz, J. (2012). Numerical modeling of melting process of thin metal film subjected to the short laser pulse. Archives of Foundry Engineering. 12(4), 105-108.

[10] Kumar, S. \& Katiyar, V.K. (2010). Mathematical modeling of freezing and thawing process in tissues: a porous media approach. Int. J. Appl. Mechanics. 2(3), 617-633.

[11] Chen, J.K. \& Beraun, J.E. (2001). Numerical study of ultrashort laser pulse interactions with metal films. Numerical Heat Transfer. Part A, 40, 1-20.

[12] Chen, G., Borca-Tascius, D. \& Yang, R.G. (2004). Nanoscale heat Transfer, Encyclopedia of NanoScience \& Nanotechnology. 7, 429-459.
[13] Zhang, Z.N. (2007). Nano/microscale heat transfer. McGraw-Hill, New York.

[14] Mochnacki, B. \& Ciesielski, M. (2015). Micro-scale heat transfer. Algorithm basing on the Control Volume Method and the identification of relaxation and thermalization times using the search method. Computer Methods in Materials Science. 15(2), 353-361.

[15] Mochnacki, B. \& Ciesielski, M. (2007). Application of Thiessen polygons in control volume model of solidification. Journal of Achievements of Materials and Manufacturing Engineering. 23(2), 75-78.

[16] Tang, D.W. \& Araki, N. (1999). Wavy, wavelike, diffusive thermal responses of finite rigid slabs to high-speed heating of laser pulses. International Journal of Heat and Mass Transfer. 42, 855-860. 